\title{
Auto-immune skin diseases in animals: time to reclassify and review after 40 years
}

Thierry Olivry ${ }^{1,2}$

\begin{abstract}
It has been more than 40 years since the dual descriptions of canine pemphigus vulgaris. Over the ensuing four decades, the reports of - mostly canine-novel autoimmune skin diseases (AISDs) have progressed in successive waves separated by long periods of quiescence. This Editorial introduces a series of comprehensive review papers on the various canine and feline AISDs. This collection of articles aims at remediating the current veterinary literature deficiency on this topic by summarizing the key historical, clinical, histological, immunological and treatment characteristics of animal AISDs.
\end{abstract}

\section{Editorial}

It has been more than 40 years since the dual descriptions of canine pemphigus vulgaris [1,2]. Over the ensuing four decades, the reports of-mostly canine-novel autoimmune skin diseases (AISDs) have progressed in successive waves separated by long periods of quiescence (Fig. 1a). After, or concurrently with these later descriptions, auto-antigen(s) were characterized following their successive discoveries in human AISDs (Fig. 1b).

At first, animal AISDs were separated into "vesiculous or bullous" (i.e., the blister-forming pemphigus and pemphigoid variants) and "non-bullous" diseases (i.e., discoid and systemic lupus erythematosus) [3]. With the recognition of vesicular cutaneous lupus erythematosus in Collie breeds and the often-non-bullous mucous membrane pemphigoid in dogs and cats, this original classification no longer seemed relevant or of any clinical value. At this time, we propose instead to create a nosology based on the dominant mechanism of lesion formation. This classification additionally provides a simple rationale for the implementation of immunosuppressive treatment regimens designed from the known mechanism of action of the various drugs.

We now suggest to separate the animal AISDs into those with lesions due-or presumed to be due-to the action of auto-antibodies (Additional file 1: Table S1) and those

\footnotetext{
Correspondence: tolivry@ncsu.edu

${ }^{1}$ Department of Clinical Sciences, College of Veterinary Medicine, North Carolina State University, Raleigh, NC 27606, USA

${ }^{2}$ Comparative Medicine Institute, North Carolina State University, Raleigh, NC, USA
}

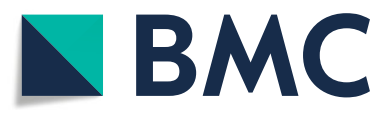

(c) The Author(s). 2018 Open Access This article is distributed under the terms of the Creative Commons Attribution 4.0 International License (http://creativecommons.org/licenses/by/4.0/), which permits unrestricted use, distribution, and

reproduction in any medium, provided you give appropriate credit to the original author(s) and the source, provide a link to the Creative Commons license, and indicate if changes were made. The Creative Commons Public Domain Dedication waiver (http://creativecommons.org/publicdomain/zero/1.0/) applies to the data made available in this article, unless otherwise stated. by-usually cytotoxic-T-lymphocytes (Additional file 2: Table S2). Within these two broad categories of AISDs, entities can be then separated logically along their main cellular or molecular targets.

While there are now many old or more recent single case reports and series of AISDs in dogs, cats, horsesand even goats, cattle, pigs, and sheep-there are no current review papers that summarize the key historical, clinical, histological, immunological and treatment characteristics of animal AISDs.

This new collection of open access articles aims at remediating this veterinary literature deficiency.

At this time, we have planned no less than six reviews on the various canine and feline AISDs. Some will have the typical format of narrative-yet in-depth-reviews (for example this first one on canine cutaneous lupus erythematosus variants), while some others will embrace systematic reviews and meta-analysis principles. Our planned series of the very rare pemphigus vulgaris will take the latter format, which will allow us to gather and regroup all of the information available in every single case report or mechanistic paper ever published on this very rare animal AISD.

Provided that this collection has the success and worldwide diffusion that we hope, we will likely expand it with additional reviews on animal immune-mediated (inflammatory) diseases (IMIDs), which we define as diseases in which a dysregulated immune-response to exogenous-but not self-antigen(s) develops or fails to abate. These diseases are not very well-characterized, and, as will be the 


\section{a Timeline of the first descriptions of canine AISDs}

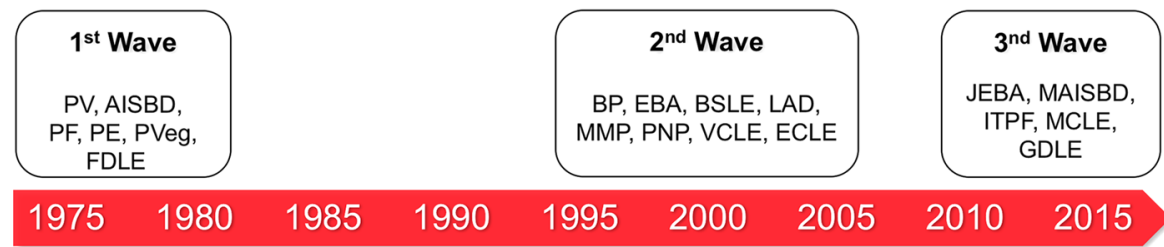

b Timeline of the characterization of the auto-antigen(s) targeted in canine AISDs

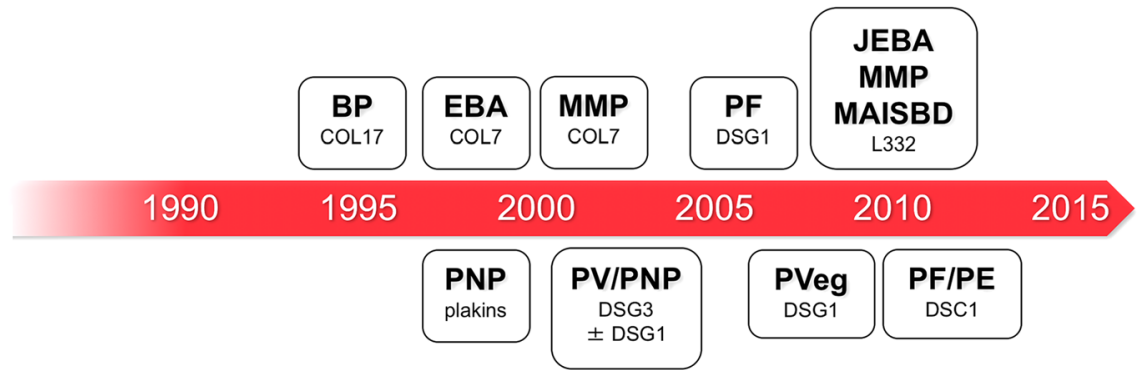

Fig. 1 Historical timelines of canine auto-immune skin diseases. a Timeline of the first descriptions of canine auto-immune skin diseases. b Timeline of the discovery of the targeted auto-antigens in the antibody-mediated auto-immune skin diseases. For abbreviations, please refer to the end of this editorial

case for erythema multiforme, Stevens-Johnson syndrome and toxic epidermal necrolysis, one would need to develop a consensus on clinical characteristics before scrutinizing and summarizing the literature in a usable format.

We hope that this journal's readership will enjoy this new collection of review articles.

\section{Additional files}

Additional file 1: Table S1. Revised classification of auto-antibodymediated auto-immune skin diseases in animals. Bolded are the most common diseases in the various species. Underlined are major autoantigens, i.e., those recognized by serum auto-antibodies in more than $50 \%$ of at least ten patients with the disease. For abbreviations, please refer to the end of this editorial. (DOCX $79 \mathrm{~kb})$

Additional file 2: Table S2. Revised classification of lymphocytemediated autoimmune skin diseases in animals. Bolded are the most common diseases in the various species. For abbreviations, please refer to the end of this editorial. (DOCX $45 \mathrm{~kb}$ )

\section{Abbreviations}

AA: Alopecia areata; AISD: Auto-immune skin disease; BP: Bullous pemphigoid; BSLE-I: Bullous systemic lupus erythematosus, type I; COL17: Collagen XVII; COL7: Collagen VII; DSC1: Desmocollin-1;

DSG1: Desmoglein-1; DSG3: Desmoglein-3; EBA: Epidermolysis bullosa acquisita; ECLE: Exfoliative cutaneous lupus erythematosus; FDLE: Facial discoid lupus erythematosus; GDLE: Generalized discoid lupus erythematosus; IgAP: IgA pemphigus; ITPF: Insecticide-triggered pemphigus foliaceus; JEBA: Junctional epidermolysis bullosa acquisita; L332: Laminin-332; LAD: Linear IgA disease; MAISBD: Mixed auto-immune subepidermal blistering dermatosis; MCLE: Mucocutaneous lupus erythematosus; MMP: Mucous membrane pemphigoid; PE: Pemphigus erythematosus; PF: Pemphigus foliaceus; PG: Pemphigoid of gestation; PNP: Paraneoplastic pemphigus; PP: Pseudopelade; PV: Pemphigus vulgaris; PVeg: Pemphigus vegetans;
SA: Sebaceous adenitis; VCLE: Vesicular cutaneous lupus erythematosus; VKH: Vogt-Koyanagi-Harada syndrome

\section{Acknowledgements}

I am grateful to Dr. Hayley Henderson, Editor of the BMC Veterinary Research, for having embraced the concept of these comprehensive reviews, for having proposed a new collection to host them.

Author's contributions

TO has written, read and approved the final manuscript.

Competing interests

The author declares that he has no competing interests.

\section{Publisher's Note}

Springer Nature remains neutral with regard to jurisdictional claims in published maps and institutional affiliations.

Received: 23 April 2018 Accepted: 23 April 2018

Published online: 11 May 2018

\section{References}

1. Stannard AA, Gribble DH, Baker BB. A mucocutaneous disease in the dog resembling pemphigus vulgaris in man. J Am Vet Med Assoc. 1975;166:575-82

2. Hurvitz Al, Feldman E. A disease in dogs resembling human pemphigus vulgaris: case reports. J Am Vet Med Assoc. 1975;166:585-90.

3. Scott DW, Wolfe MJ, Smith CA, Lewis RM. The comparative pathology of non-viral bullous skin diseases in domestic animals. Vet Pathol. 1980;17:257-81. 